\title{
Effect of transverse plane alterations in footwear design on lower limb biomechanics during running.
}

\begin{abstract}
Background

Abduction of the forefoot, relative to the rearfoot, is a component of rearfoot pronation. It is therefore feasible that an ill-fitting shoe, which creates a relative abduction of the forefoot, could influence rearfoot motion with a corresponding effect on transverse plane tibial rotation.
\end{abstract}

$\underline{\text { Aim }}$

To investigate whether transverse plane motion of the tibia is altered with specially designed footwear in which the front of the shoe is positioned either laterally or medially.

\section{Methods}

Kinematic data was collected from $n=9$ participants during running at $3.9 \mathrm{~m} / \mathrm{s}$ in three footwear conditions: baseline, lateral forefoot and medial forefoot. ANOVA testing was then used to understand whether the different shoe designs affected tibial and/or pelvic motions.

\section{$\underline{\text { Results }}$}

There was marked inter-subject variability in response to the two designs. Whereas some participants responded to the lateral design with a decrease in tibial rotation, others responded with a clear increase in external transverse tibial rotation. However, the mean effect was small and there were no significant difference in either the transverse tibial angle at initial contact or the transverse tibial range of movement. Interesting, the footwear designs appeared to have an effect on frontal plane pelvic motion. However, in some subjects pelvic motions were increased, whereas in others they were decreased, again leading to a non-significant mean effect.

\section{Conclusion}

These results may support the idea that an ill-fitting running shoe could lead to alterations in lower limb kinematics; however further work is required to understand individual differences in response. 